\title{
Medium-term follow up of residual shunting and potential complications after transcatheter occlusion of the ductus arteriosus
}

\author{
Alan G Magee, Oliver Stumper, Janet E Burns, Michael J Godman
}

\begin{abstract}
Objectives-To determine the causes and outcome of residual shunting after transcatheter occlusion of persistent ductus arteriosus with the Rashkind double umbrella occluder, and to determine the potential of the device to produce obstruction to flow in the aorta and left pulmonary artery.

Design-Angiographic examination of morphology of ductus followed by prospective clinical and ultrasound evaluation (including cross sectional imaging, colour flow mapping, and pulse wave Doppler) of all patients undergoing occlusion of persistent ductus arteriosus between October 1987 and July 1992.

Patients-140 patients with ages between 0.5 and 78 (median 3.8) years and weights between $6 \cdot 8$ and 74 (median 13.8) kg.

Interventions-Attempted implantation of the Rashkind double umbrella ductus occluder under angiographic control through a transvenous $(n=136)$ or transarterial $(n=4)$ approach.

Main outcome measures-Successful occlusion of ductus; frequency, pattern, and prognosis of residual shunts; Doppler velocities in left pulmonary artery and aorta; volume loading of the left heart.
\end{abstract}

Results-Including reocclusions the overall rate of successful occlusion was $96 \%$. A total of six devices embolised at the time of operation $(4 \cdot 3 \%)$ with no sequelae. There were no anatomical factors that predicted a poor outcome, but suboptimal positioning of the device led to a significantly higher incidence of residual shunts $(p<0 \cdot 001)$. Colour flow mapping correctly identified shunts that were unlikely to close spontaneously ( $n=$ 9) and to date seven have undergone successful closure with a second device. Encroachment of device legs produced statistically ( $p<0.001$ ) but not clinically significant increases in left pulmonary artery Doppler velocities that diminished with time.

Conclusions-Transcatheter occlusion provides a safe and effective means of closing a persistent ductus arteriosus. Doppler colour flow mapping is necessary for follow up and shows those ducts requiring reocclusion. The device did not produce significant disturbance to flow in the pulmonary arteries or aorta.

(Br Heart F 1994;71:63-69)

Surgery for congenital heart disease began with the first successful ligation of a ductus arteriosus by Gross and Hubbard in $1938 .{ }^{1} \mathrm{~A}$ non-surgical catheter occlusion technique was pioneered by Porstman et al in 1967, with a plastic foam plug delivered along an arteriovenous loop. ${ }^{2}$ Although successful in some patients, this system never gained widespread acceptance because of its complexity and the large size of the delivery catheter. In 1979 Rashkind and Cuaso described successful duct occlusion in a $3.5 \mathrm{~kg}$ infant, with a single umbrella consisting of a foam covered stainless steel prosthesis that attached itself to the ductus by means of hooks. ${ }^{3}$ This prototype led to the development of a $12 \mathrm{~mm}$ double umbrella device, that with certain modifications and the introduction of a larger $17 \mathrm{~mm}$ size, has increasingly been adopted as an alternative to surgery in many centres. ${ }^{4-6}$

Early and intermediate term results for the umbrella device indicate rates of successful occlusion of the duct ranging from $66 \%$ to $92 \%$ with a low incidence of complications. ${ }^{4-6}$ Whether or not transcatheter occlusion becomes totally accepted as the treatment of choice compared with surgery, ${ }^{7}$ will be determined by the frequency of residual shunting at late follow up and the frequency of catheter or device induced complications that have been reported as including device embolisation, haemolysis, and endarteritis. ${ }^{4-6}$

In this study we review our experience of transcatheter occlusion between 1987 and 1992, with particular reference to (a) the frequency of complete occlusion after delivery of the device, $(b)$ the frequency, mode, and causes of residual shunting, and $(c)$ the potential of the device to produce obstruction to flow in the aorta and left pulmonary artery.

\section{Patients and methods}

PATIENTS

Between October 1987 and May 1992 we have attempted closure of a ductus arteriosus in 140 patients with the Rashkind double umbrella occluder. Ages were from 0.5 to 78 (median 3.8) years and weights ranged from 6.8 to 74 (median 13.8) $\mathrm{kg}$. The group included 12 adult patients (over the age of 16). There were 52 male patients and 88 
female patients. One patient had a small muscular ventricular septal defect and one had rheumatic heart disease with pulmonary hypertension; the remainder had an isolated ductus. Diagnosis was based on clinical examination and confirmed by echocardiography and subsequent cardiac catheterisation.

\section{OCCLUSION TECHNIQUE}

The procedure was performed with the previously described transvenous technique ${ }^{8}$ in all but four cases in whom a transarterial technique was used after unsuccessful attempts at occlusion from the venous side. General anaesthesia was used in all children and in two of the adults. Aortograms before occlusion were performed through a 5 French pigtail catheter in children and 6 or 7 French in adults. The shape of the duct and diameter at the pulmonary artery end were assessed. The $12 \mathrm{~mm}$ device was selected for a ductus with internal diameter less than $4 \mathrm{~mm}$ and the $17 \mathrm{~mm}$ device for those measuring greater than $4 \mathrm{~mm}$. For six months during the study the $12 \mathrm{~mm}$ device was in short supply and 18 patients with a ductus from 3-4 $\mathrm{mm}$ who might otherwise have had a $12 \mathrm{~mm}$ device, had implantation of a $17 \mathrm{~mm}$ device. Ducts larger than $9-10 \mathrm{~mm}$ in diameter were generally considered too large for catheter occlusion. The device was washed in saline to evacuate micro air bubbles from the foam. No heparin was given before implantation. Since 1989 antibiotic cover $(25 \mathrm{mg} / \mathrm{kg}$ cefuroxime) has been given at the time of implantation of the device. A repeat aortogram was performed in most cases after release of the device to assess the adequacy of closure. On day one all patients were examined clinically and by cross sectional and Doppler ultrasound; since 1988 this has also included colour flow mapping.

FOLLOW UP EXAMINATIONS

Patients were reviewed about four to six

Figure 1 Lateral angiograms showing $(A)$ typical funnel shaped ductus and $(B)$ device in position with hinge point at the pulmonary artery end, distal limbs nicely flexed and no residual shunting. weeks after implantation and then at six monthly intervals. Occlusion was assumed to occur just before the first ultrasound examination showing absence of residual flow. If secure closure was shown follow up was at yearly intervals after the first six months. Since becoming available colour flow mapping has been carried out at the time of clinic visits in all patients. Mean duration of follow up is $2 \cdot 3$ years (range $0 \cdot 1$ to five).

\section{REVIEW OF ANGIOGRAMS}

Angiograms were reviewed before most of the follow up examinations, but the reviewers were not completely unaware of results as one of us (MJG) was involved in all implantations. With the lateral angiogram, the shape of the ductus was assessed and the narrowest internal diameter measured (usually the pulmonary artery end). The shape of the ductus was classified into (a) funnel (a wide aortic ampulla tapering to a narrow pulmonary end, fig 1); (b) tubular (fig 2); (c) nipple; (d) window (short tubular, fig 3 ), and (e) tortuous.

Sizes were grouped into $<2 \mathrm{~mm}, 2$ to $<4 \mathrm{~mm}, 4$ to $<6 \mathrm{~mm}$, and $>6 \mathrm{~mm}$ (fig 2 ).

After occlusion, the adequacy of positioning of the device and the degree and direction of any residual shunts were assessed. The relation of the hinge point of the device to the narrowest area of the ductus and the relation of the legs of the distal umbrella to the aortic ampulla were used to assess the adequacy of positioning of the device. An attempt was made to quantify any residual leak of contrast by noting if it was a diffuse blush or a jet, and if a jet, whether or not it filled the main pulmonary artery.

\section{ECHOCARDIOGRAPHIC AND DOPPLER}

ASSESSMENTS

Studies were performed with a Toshiba SSH160A or SSH140A with both 3.5 and 5 $\mathrm{MHz}$ transducers. A ductal view ${ }^{9}$ and a high left parasternal view with clockwise rotation were used to visualise the pulmonary arteries

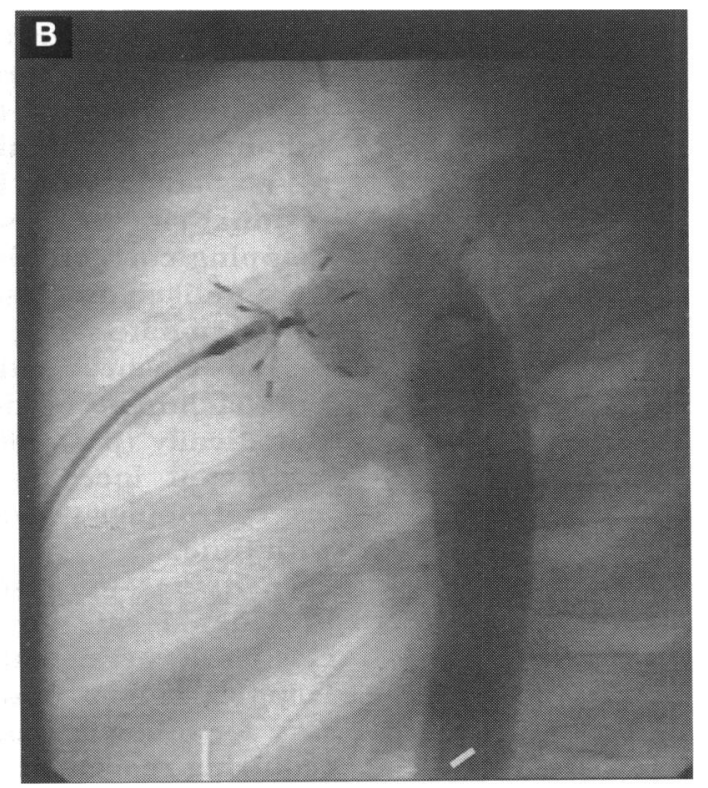


Figure 2 (A) Large parallel or tubular ductus with internal diameter $7 \mathrm{~mm}$ and (B) $17 \mathrm{~mm}$ device in position with a superior limb of the distal prongs not well flexed and protruding on the aortic side. Hinge point poorly related to pulmonary artery end of the ductus.
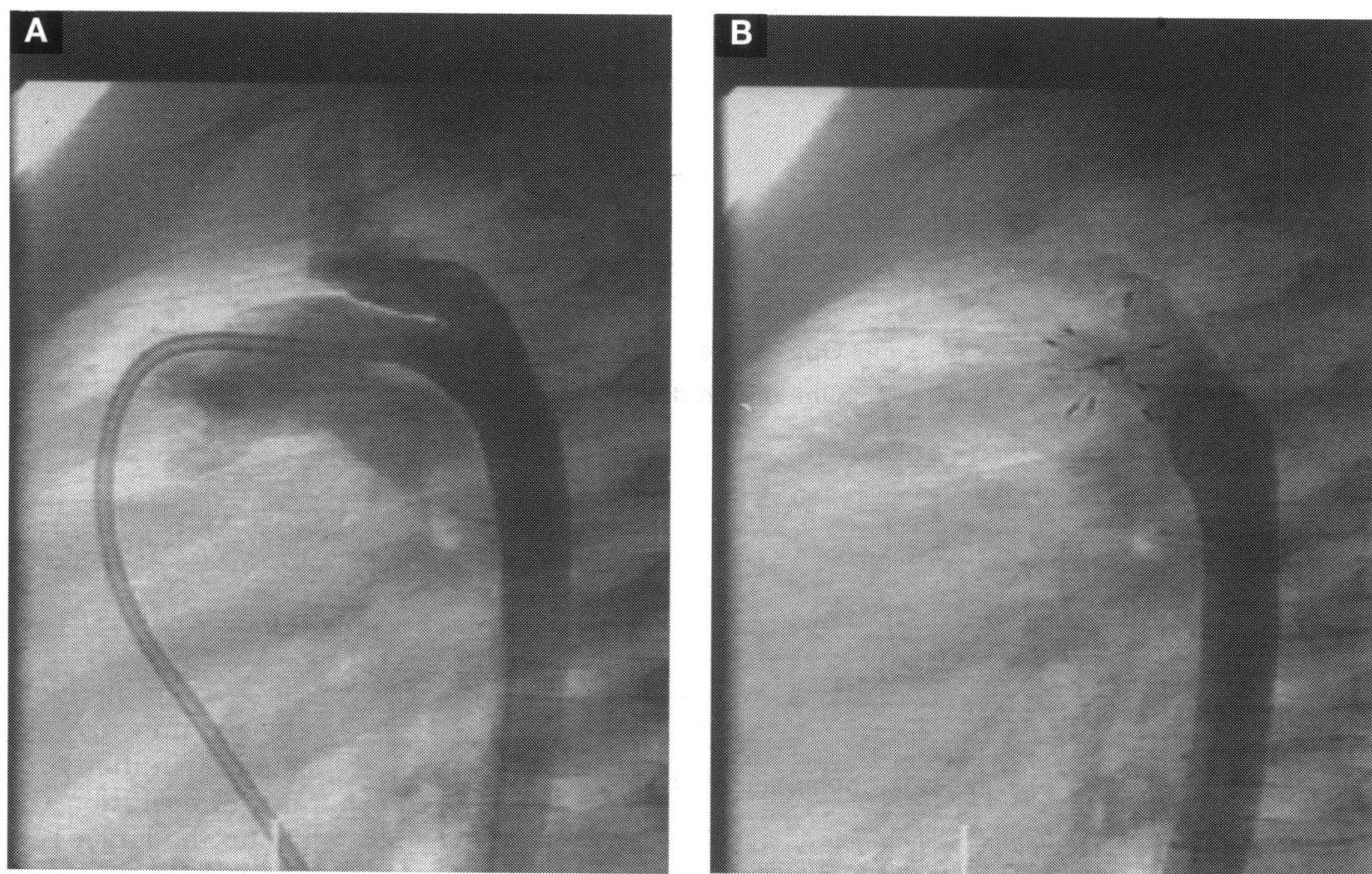

Figure 3 (A) Short window shaped ductus and (B) device hinge point after occlusion well anterior to pulmonary artery end of the ductus and angled inferiorly. Device protruding noticeably into pulmonary artery.
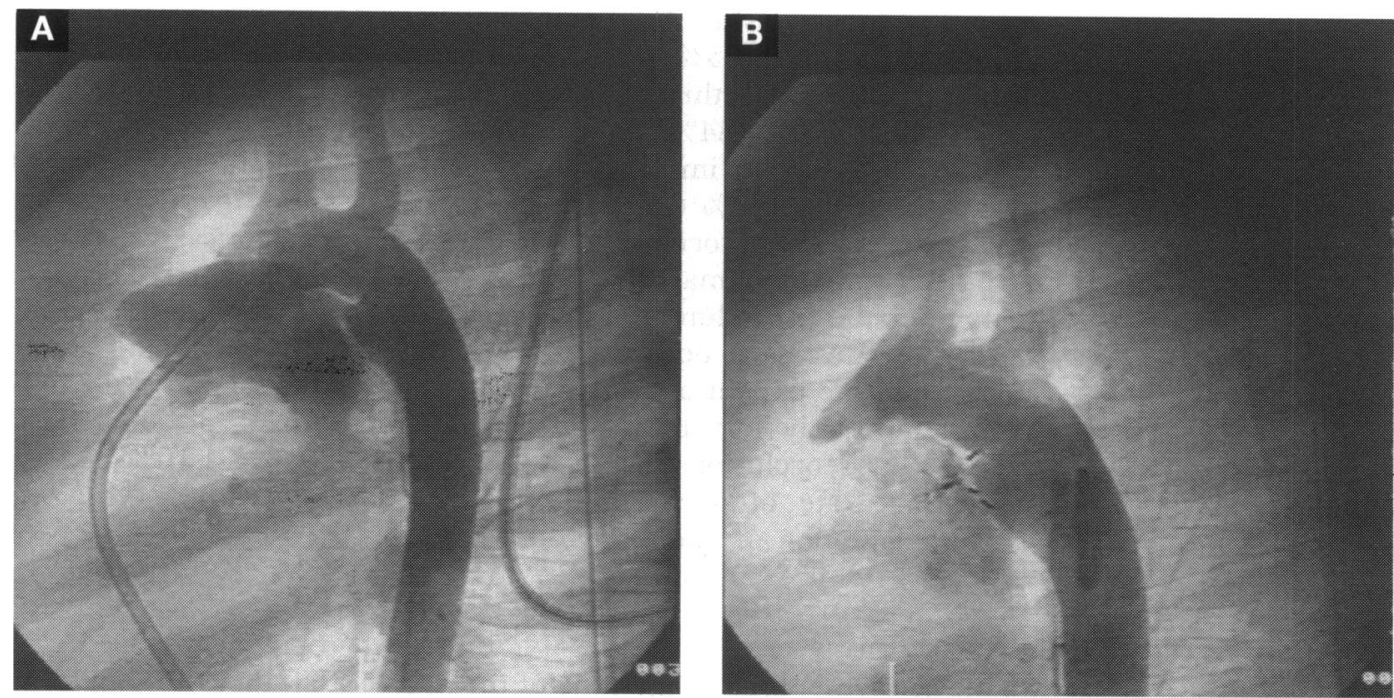

and the device. The suprasternal view was also used to assess the pattern of flow and velocity in the descending aorta. The transducer was moved through multiple planes to ensure that no leak was missed. Pulsed and continuous wave Doppler spectral traces were obtained of the main, left, and right pulmonary arteries and descending aorta, guided by the colourflow map. The direction, velocity, and width of any residual shunts were assessed, as well as whether flow was continuous or discontinuous. Protrusion of the device and any increase in Doppler velocity were assessed across the left pulmonary artery and descending aorta. In 50 cases, velocity across the left pulmonary artery was measured before and after implantation, and in the rest velocity after implantation was compared with velocity across the right pulmonary artery. Also $\mathrm{M}$ mode measurements of left atrial diameter and aortic root diameter were recorded from the parasternal long axis views to assess volume loading of the left heart.

\section{STATISTICS}

Results are expressed as mean with 95\% confidence intervals (95\% CIs). Kaplan Meier product limit analysis, the logrank test, and Student's $t$ test were used to compare data between groups. In the Kaplan Meier analysis of rates of occlusion, patients with residual shunts are entered as "censored" at the time of latest follow up that showed residual flow or at the time of implantation of a second device.

\section{Results}

OVERALL OCCLUSION RATE

We attempted to occlude the ductus of 140 patients with a single device. Early in the series the procedure was abandoned in two cases. In the first, a patient under sedation had an uncontrollable cough and in the second the Mullins sheath could not be advanced beyond the right ventricular outflow tract. Further attempts after 12 months in the first case and nine months in the 


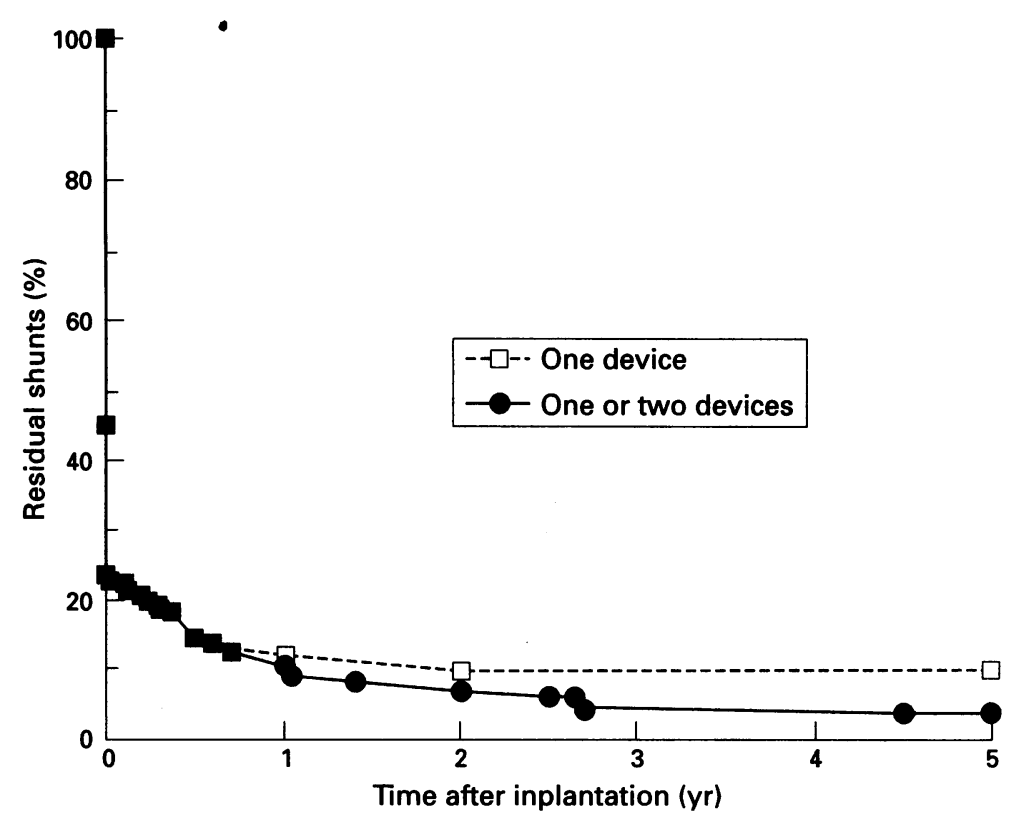

Figure 4 Rates of residual shunting against time after implantation of one device or after implantation of one or two devices (including reocclusions).

second were successful. Angiography showed occlusion in $55 \%$ (95\% CI $47 \%-63 \%)$; on ultrasound at day 1 this value had risen to $77 \%$ (95\% CI $70 \%-84 \%)$ and the occlusion rate at follow up after implantation of a single device (fig 4) was $90 \%$ (95\% CI $85 \%-95 \%$ ). There were 21 late occlusions shown with Doppler colour flow mapping from $0 \cdot 02-2 \cdot 0$ years after the procedure. All late occlusions were shown to have occurred by 0.7 years apart from one patient at two years who had been temporarily lost to follow up. There were seven reocclusions all of which were successful. Rate of occlusion at follow up after implantation of one or two devices was 96\% (95\% CI 93\%-99\%).

\section{COMPLICATIONS}

Six devices embolised at the time of the operation $(4 \cdot 3 \%)$. Four were retrieved through the catheter and in three of these four a further device was inserted uneventfully; the remaining girl had considerable pulmonary hypertension and it was decided to close her ductus surgically. In a frail 63 year old woman with rheumatic heart disease and pulmonary hypertension, occlusion was made technically difficult by poor imaging. The device was placed but $x$ ray films 24 hours after the operation showed that the device had migrated to the bifurcation of the aorta. As blood supply to the lower limbs was not compromised it was judged prudent not to attempt removal. In a further child, it was not

Table 1 Numbers in each category of the narrowest internal diameter of the ductus together with rates of residual shunting seen on angiograms after release of device, on ultrasound at day 1 and at latest follow up

\begin{tabular}{llllll}
\hline Sizes $(\mathrm{mm})$ & $0-2$ & $2-4$ & $4-6$ & $>6$ & $p$ Value $^{*}$ \\
\hline No & 30 & 71 & 20 & 4 & \\
Angiogram (\%) & 45 & 51 & 62 & 75 & $\mathrm{p}>0.2$ \\
Ultrasound (\%) & 10 & 31 & 31 & 75 & $\mathrm{p}>0.2$ \\
Follow up (\%) & 5 & 10 & 31 & 37.5 & $0.1<\mathrm{p}>0.05$ \\
\hline
\end{tabular}

${ }^{\star} \mathrm{p}$ Values refer to the probability of a significant difference in the rates of residual shunting or occlusion between categories. possible to retrieve the device from the distal right pulmonary artery. The device was removed through a right thoracotomy and the ductus was surgically ligated one week later. The two surgically treated children were "censored" in the analysis of residual shunting at the time of surgery, and the elderly woman is still considered to have a shunt. There were no cases of haemolysis or endarteritis.

INFLUENCE OF DUCTUS SIZE AND SHAPE, DEVICE SIZE, AND POSITIONING ON RATES OF OCCLUSION

High quality lateral angiograms were available for analysis in 125 cases.

\section{DUCTUS DIAMETER}

Narrowest diameters were $<2 \mathrm{~mm}$ in $24 \%$, 2 to $<4 \mathrm{~mm}$ in $56.6 \%, 4$ to $6 \mathrm{~mm}$ in $16.4 \%$, and $>6 \mathrm{~mm}$ in $3 \%$ (table 1 ). Size made no significant difference to immediate or day 1 rates of occlusion (both $p>0 \cdot 2$ ), but at follow up the larger two categories were slightly less likely to show occlusion $(0 \cdot 1<\mathrm{p}>0 \cdot 05)$.

\section{DUCTUS SHAPES}

These were grouped into funnel $(65 \cdot 6 \%)$, tubular $(12 \cdot 3 \%)$, nipple shaped $(8 \cdot 2 \%)$, window $(5 \cdot 7 \%)$, and tortuous $(8 \cdot 2 \%)$. As we thought that successful occlusion might be more likely in the funnel and nipple shaped categories, we compared these groups with the others. The rates of residual shunting (fig 5) on angiography were $40 \%$ (95\% CI $30 \%-50 \%$ ) for funnel and nipple shaped ducts and $56 \%(95 \%$ CI $39 \%-73 \%)$ for the others $(p>0.1)$; on ultrasound at day $118 \%$ (95\% CI 10\%-26\%) and 34\% (95\% CI 18\%$50 \%)(\mathrm{p}>0.1)$; and at follow up $7.26 \%$ (95\% CI $2 \%-12 \cdot 6 \%)$ and $17 \cdot 2 \%$ (95\% CI $4 \%-28 \%)(\mathrm{p}=0 \cdot 1)$.

DEVICE SIZE

Of the 140 single implantations we used a total of $8212 \mathrm{~mm}$ devices and $5817 \mathrm{~mm}$ devices (fig 6). The rates of residual leaks on angiography were $30 \%$ (95\% CI $20 \%-40 \%$ ) for $12 \mathrm{~mm}$ devices and $63.5 \%(95 \% \mathrm{CI}$ $50.5 \%-76.5 \%)$ for $17 \mathrm{~mm}$ devices $(\mathrm{p}=0.01)$, and on ultrasound at day $113.7 \%$ (95\% CI $6 \%-21.5 \%$ ) for $12 \mathrm{~mm}$ devices and $35 \%$ (95\% Cr $22 \%-48 \%$ ) for $17 \mathrm{~mm}$ devices $(p=0.01)$. The follow up rates of $6 \cdot 1 \%(95 \%$ CI $1 \%-11 \%$ ) for $12 \mathrm{~mm}$ devices and $15.4 \%$ (95\% CI $6 \cdot 1 \%-24 \cdot 7 \%$ ) for $17 \mathrm{~mm}$ devices were not significantly different $(p=0 \cdot 1)$. As already mentioned, 18 patients with a ductus diameter measuring $<4 \mathrm{~mm}$ had $17 \mathrm{~mm}$ devices implanted. Rates of residual shunting seen on angiograms were $78 \%$ compared with $30 \%$ for appropriately treated small ducts ( $p<0.001$ ); after 24 hours 33\% v 13.7\% $(\mathrm{p}=0.05)$; and at follow up $5.5 \%$ v $6.1 \%$ $(\mathrm{p}>0 \cdot 1)$.

\section{DEVICE POSITION}

According to the above criteria device position was suboptimal on a total of 12 occasions (figs 2 and 3). A suboptimal position led to significantly poorer rates of occlusion 


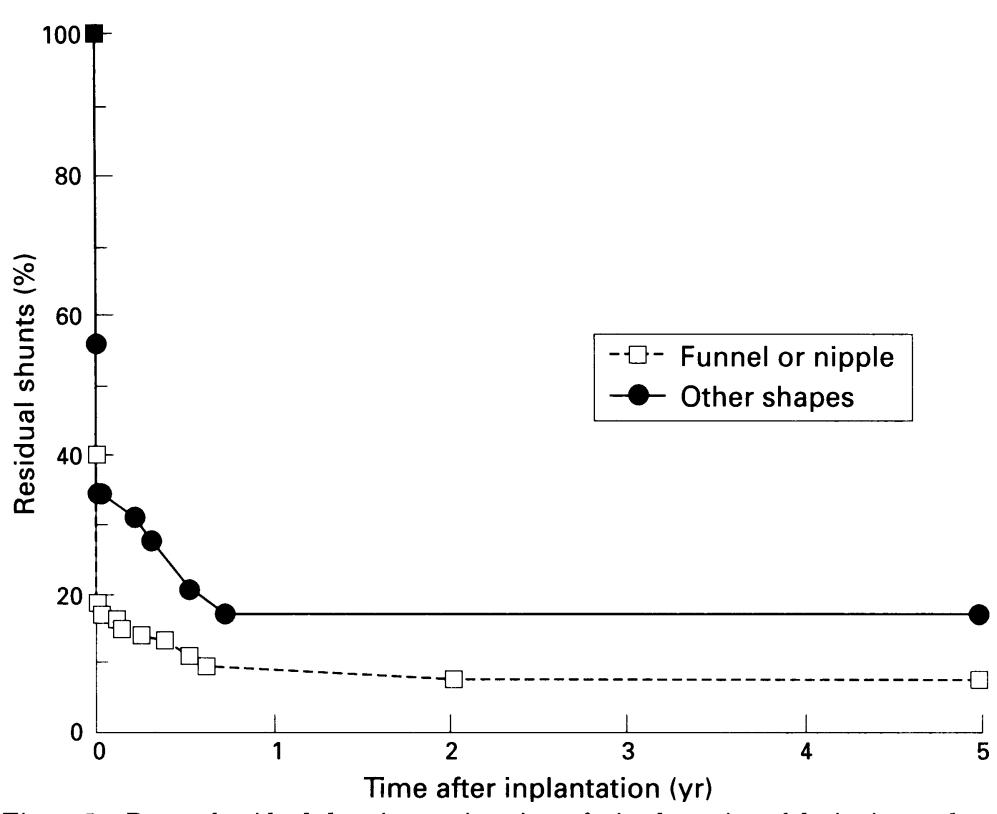

Figure 5 Rates of residual shunting against time afte implantation of device into a ductus with a funnel or nipple shape compared with residual shunting after implantation of device into a ductus with a tubular, window, or tortuous shape.

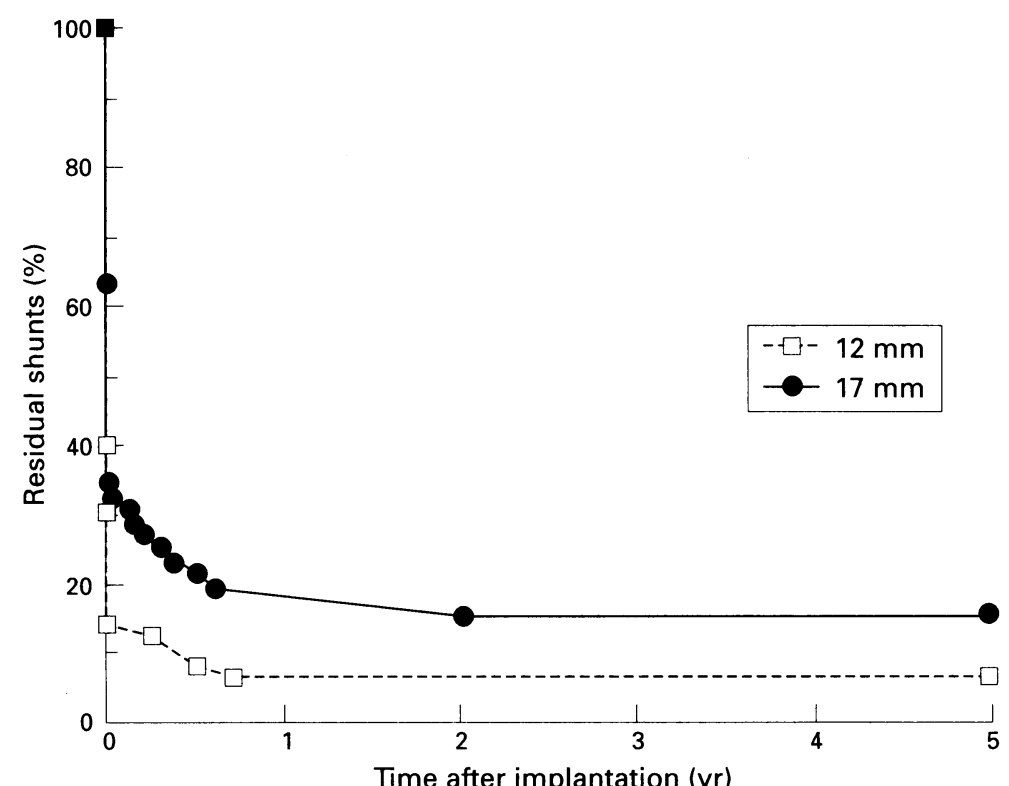

Figure 6 Rates of residual shunting against time after implantation of $12 \mathrm{~mm}$ or $17 \mathrm{~mm}$ devices.

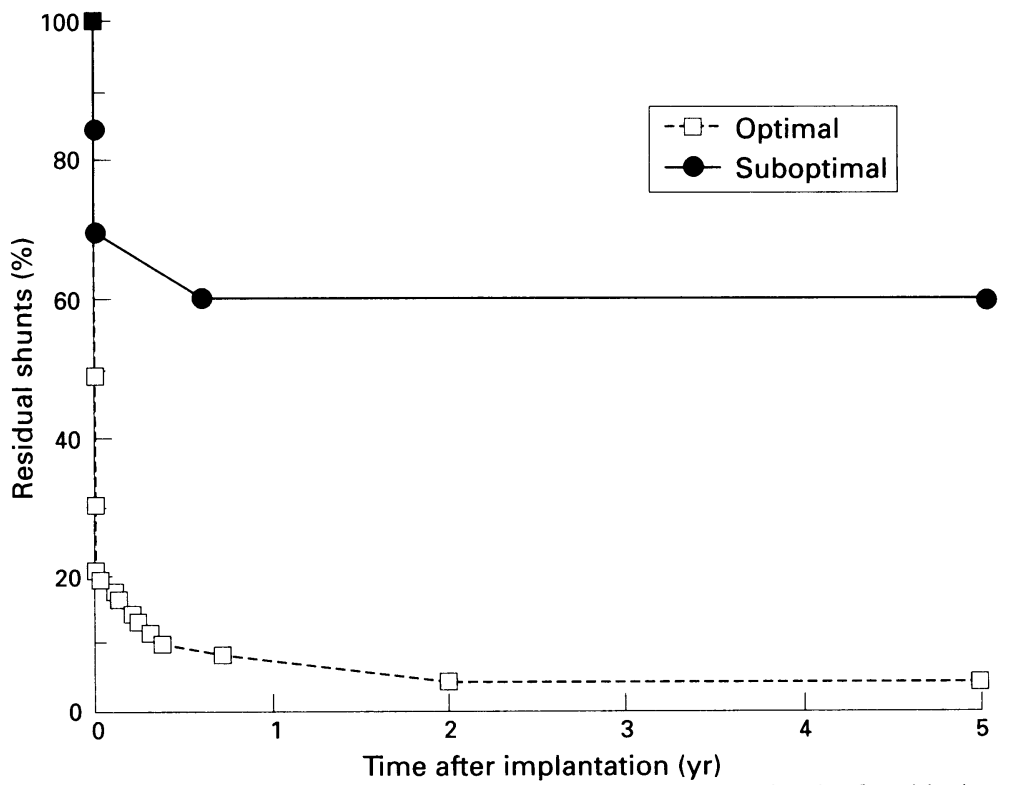

Figure 7 Rates of residual shunting against time after optimal or suboptimal positioning of device. from the time of implantation onwards (fig 7 $\mathrm{p}<0.001)$.

OUTCOME OF RESIDUAL SHUNTS SEEN ON ANGIOGRAMS

Residual shunts seen on angiograms were divided into three groups: (a) small diffuse blushes of contrast leaking through the device ( $\mathrm{n}=36)$, (b) small jets not filling the main pulmonary artery $(n=13)$, and $(c)$ large jets filling the main pulmonary artery $(n=11)$.

Patients in group 1 had a good prognosis for closure with 25 closed by 24 hours. Ten showed late closure and one developed into a low velocity flame on colour flow mapping (see next section). In group 2 small jets were superior to the device in 10 patients and inferior in three. Four had closed by day 1 , four occluded late, and five have not occluded at latest follow up. In group 3 large jets were superior in nine patients and in two both above and below the device. One closed overnight, two closed late, and eight have not occluded at latest follow up.

\section{FOLLOW UP OF RESIDUAL SHUNTS WITH} DOPPLER COLOURFLOW MAPPING

Four types of residual shunt were seen:

(a) a broad continuous high velocity jet reaching to the pulmonary valve and associated with a continuous murmur $(n=9)$. Seven have had a second device after a mean of two (range 1-4.5) years, and the remaining two have continuous flow at latest follow up.

(b) A narrow non-continuous high velocity jet $(n=21)$. Sixteen have closed, two have developed into low velocity flames (see $(d)$ ), and three remain present.

(c) Colour around the device not extending into the pulmonary artery $(n=4)$; all disappeared with follow up. In three out of the four there had not been a leak of contrast on the angiogram after implantation.

(d) Low velocity late systolic flames with Doppler velocity $<2 \mathrm{~m} / \mathrm{s} \quad(\mathrm{n}=2)$; both present 1.6 and 1.7 years after operation.

\section{DOPPLER VELOCITY IN LEFT PULMONARY} ARTERY AND AORTA

Table 2 shows the increases in Doppler velocity across the origin of the left pulmonary artery and along the descending aorta before and after implantation of a single 12 or $17 \mathrm{~mm}$ device. There were statistically significant increases in velocity across the left pulmonary artery after implantation of either device but no significant increase in the descending aorta. The mean maximum velocity in the left pulmonary artery was 1.205 (range $0.75-2) \mathrm{m} / \mathrm{s}$ after the smaller device and $1 \cdot 28$ (range $0 \cdot 6-2 \cdot 1$ ) $\mathrm{m} / \mathrm{s}$ after the larger device. In $38 \%$ of cases there was no increase in velocity after implantation of a $12 \mathrm{~mm}$ device, and in $25 \%$ of cases there was no increase after a $17 \mathrm{~mm}$ device.

After reocclusion $(n=7)$ there was an increase across the left pulmonary artery from zero to a mean of $0.5(95 \%$ CI $0.3-0.7) \mathrm{m} / \mathrm{s}$ $(p=0.004)$. The mean maximum velocity was 1.6 (range $1-2) \mathrm{m} / \mathrm{s}$. 
Table 2 Mean (95\% CI) increases in Doppler velocity across the origin of the left pulmonary artery (LPA) and across the descending aorta (DAo) before and after implantation of either $12 \mathrm{~mm}$ or $17 \mathrm{~mm}$ devices

\begin{tabular}{lllll}
\hline Site & Device & $\begin{array}{l}\text { Increase before } \\
(\mathrm{m} / \mathrm{s})\end{array}$ & $\begin{array}{l}\text { Increase after } \\
(\mathrm{m} / \mathrm{s})\end{array}$ & $p$ Value $^{*}$ \\
\hline LPA & $12 \mathrm{~mm}$ & $0.03(0-0.06)$ & $0.29(0 \cdot 15-0.4)$ & $<0.001$ \\
& $17 \mathrm{~mm}$ & $0.01(0-0.03)$ & $0.28(0 \cdot 2-0.36)$ & $<0.001$ \\
DAo & $12 \mathrm{~mm}$ & 0 & $0.03(0-0.05)$ & 0.2 \\
& $17 \mathrm{~mm}$ & 0 & $0.05(0-0.12)$ & 0.13 \\
\hline
\end{tabular}

${ }^{\star} \mathrm{p}$ Values by paired Student's $t$ test. date five of these have required a second device. Malposition alone does not necessarily mean that a second device will be needed but taken with the presence of a continuous murmer there is a high likelihood that one will be required. If the aortogram before release of the device indicates a poor position of the device an attempt is now made to withdraw or advance the device slightly with the rod of the delivery catheter.

Many small residual blushes or jets of contrast were seen on angiography at the end of the procedure but in over $70 \%$ of these cases Doppler colour flow mapping showed complete occlusion by 24 hours. Colour flow Doppler is of great value in the follow up of residual shunts. The pattern of such shunts can often predict outcome, with colour around the device not extending into the pulmonary artery and discontinuous high velocity shunts indicating an excellent prognosis for closure. As reported for previous series, spontaneous closure of residual shunts may occur many months after the procedure. ${ }^{56}$ Continuous Doppler flow at six months, in our experience, usually means that a second device will be required. Angiograms in these cases had shown large jets filling the pulmonary arteries and there were continuous murmurs on auscultation.

One interesting appearance on colour flow mapping was of a low velocity flame that persisted on follow up. Whether or not prophylaxis for subacute bacterial endocarditis should continue for these cases is at present unclear. With such low velocities a negligible risk of endarteritis might be expected. Recent studies have shown fairly high rates of residual ductal flow by colour flow mapping after surgical ligation, ${ }^{7}$ and there is no reported incidence of endarteritis in this group or in the clinically silent ductus. ${ }^{1011}$ The results of this and other studies point to an extremely low incidence of endarteritis after implantation. The only case described since antibiotics were routinely given at the time of implantation was in a patient with a clearly heard residual continuous murmur who was not taking endocarditis prophylaxis. ${ }^{10}$ We cannot assume, however, that the risk of endarteritis is abolished, even after successful ductal closure until long-term follow up data is available, and now most would agree that any residual leak past a device merits continued antibiotic prophylaxis.

There has been concern regarding encroachment into the left pulmonary artery and descending aorta by the limbs of the device. ${ }^{12}$ This series showed significant increases in Doppler velocity across the origin of the left pulmonary artery caused by the limbs of both the 12 and $17 \mathrm{~mm}$ devices. Neither device caused any significant overall increase in Doppler velocity in the descending aorta. These findings contrast with those of Ottenkamp et al who found more flow disturbances in the aorta than the left pulmonary artery. ${ }^{13}$ Reocclusion with either device does seem to cause an increased Doppler velocity in the left pulmonary artery but numbers are device to the narrowest point of the ductus and the relation of the deployed distal limbs to the aortic ampulla. According to this definition 12 devices were malpositioned and to 
small. Overall there were only four cases with a Doppler velocity $\geqslant 2 \mathrm{~m} / \mathrm{s}$ the maximum being $2 \cdot 2 \mathrm{~m} / \mathrm{s}$, and no cases of occlusion or coarctation. Serial estimation of left pulmonary artery velocity seems to show that it falls with time possibly due to endothelialisation of the device and growth. To be completely sure that the device does not cause reduced flow to the left lung radioisotope scanning would be required.

Although the presence of a continuous murmur after occlusion correlates well with ultrasound findings, the presence of a murmur in systole is less helpful and some residual shunts are inaudible. A soft systolic murmur may be associated with a small shunt, a mild haemodynamic disturbance, or may have no identifiable cause.

This study further establishes transcatheter occlusion as a successful mode of treatment for the ductus arteriosus with a low incidence of complications. Overall success rate was $96 \%$ including reocclusions. Continuous residual flow on colour flow mapping by about six months identified the need for second devices that were implanted without difficulty. The presence of a device causes significant increases in Doppler flow velocities in the left pulmonary artery but not in the descending aorta. Few values reached $2 \mathrm{~m} / \mathrm{s}$ and increased velocity does seem to diminish with time. Follow up for up to five years identified no clinically significant incidence of obstruction to flow in the left pulmonary artery or aorta.
Dr Magee is the recipient of a grant from the Scottish Chest, Heart, and Stroke Association.

1 Gross RE, Hubbard JP. Surgical ligation of a patent ductus arteriosus: report of first successful case. $\mathcal{F} A M A$ 1939;112:729-31.

2 Porstmann W, Wierny L, Warnke H, Gerstberger G, Romaniuk PA. Catheter occlusion of patent ductus arteriosus, 62 cases treated without thoracotomy. Radiol Clin North Am 1971;9:203-18.

3 Rashkind WJ, Cuaso CC. Transcatheter closure of paten ductus arteriosus: successful use in a $3.5 \mathrm{~kg}$ infant. Pediatr Cardiology 1979;1:3-7.

4 Rashkind WJ, Mullins CE, Hellenbrand WE, Tait MA. Non-surgical closure of patent ductus arteriosus: clinical application of the Rashkind PDA occluder system. Circulation 1987;75:583-92.

5 Tynan $M$, et al. Transcatheter occlusion of the persistent arterial duct: report of the European registry. Lancet arterial duct: rep

6 Hosking MCK, Benson LN, Musewe N, Dyck JD, Freedom RM. Transcatheter occlusion of the persistently patent ductus arteriosus: forty month follow-up and prevalence of residual shunting. Circulation 1991; 84:2313-7.

7 Sorensen K, Kristensen B, Hansen OK. Frequency of occurrence of residual ductal flow after surgical ligation by colour-flow mapping. Am $f$ Cardiol 1991;67 $653-4$

8 Bash SE, Mullins CE. Insertion of patent ductus arteriosus occluder by transvenous approach: a new technique [abstract]. Circulation 1985;70(suppl II):285.

9 Smallhorn JF, Huhta JC, Anderson RH, Macartney FJ Suprasternal cross-sectional echocardiography in assessment of patent ductus arteriosus. Br Heart $\mathcal{f} 1982$; 48:321-30

10 Latson LA. Residual shunts after transcatheter closure of patent ductus arteriosus: a major concern or benign

11 Houston AB, Gnanapragasam JP, Lim MK, Doig WB, Coleman EN. Doppler ultrasound and the silent ductus arteriosus. Br Heart f 1991;65:97-9.

12 Smythe JF, Benson LN, Musewe N, Moes CAF, Burrows $\mathrm{P}$, Freedom RM. Left pulmonary artery morphology post ductal occlusion [abstract]. Clin Invest Med 1990; 13:C79.

13 Ottenkamp J, Hess J, Talsma MD, Buis-Liem TN Protrusion of the device: a complication of catheter occlusion of patent ductus arteriosus. Br Heart $\mathcal{f} 1992$ 68:301-3. 